\title{
Ferroelectric liquid crystals in high magnetic fields
}

\author{
Th. Rasing ${ }^{a}$, I. Muševič ${ }^{b}$, B. Žekšs $\check{~}^{b}$, R. Blinc ${ }^{b}$ and A. Seppen ${ }^{c}$ \\ "Research Institute for Materials and High Field Magnet Laboratory, University of Nijmegen, Toernooiveld, \\ 6525 ED Nijmegen, The Netherlands \\ 'J. Stefan Institute, University of Ljubljana, Jamova 39, 61111 Ljubljana, Slovenia (Yugoslavia) \\ "Philips Research Laboratories, $5600 \mathrm{JA}$ Eindhoven. The Netherlands
}

\begin{abstract}
Ferroelectric liquid crystals represent a class of materials that are fascinating from a fundamental point of view, and show a high potential for application in liquid crystal based electro-optic devices. In particular, in the presence of an external magnetic field, they display a very interesting phase diagram, where a re-entrant phase, Lifshitz point and band structure of phason excitations can be observed. Due to the strong electro-optical and magneto-optical effects of these materials, optical experiments appear to be extremely suitable and straightforward to study both the static and dynamic properties of these fascinating phases of matter.
\end{abstract}

\section{Introduction}

In the ferroelectric $\mathrm{Sm} \mathrm{C}^{*}$ liquid crystalline phase [1] rod-like molecules with a transverse electric dipole moment are arranged in layers. The distribution of molecular centers of gravity is random within the layer and thus each layer can be considered as a two-dimensional liquid. The director $n(\boldsymbol{r})$ describing the time averaged direction of the long molecular axis is tilted with respect to the layer normal at an angle $\theta$ and precesses with a period $p$ as one moves along the layer normal. The $\mathrm{Sm} \mathrm{C}^{*}$ liquid crystalline phase is thus a spatially inhomogeneous, helicoidal phase with the helical axis along the layer normal. The helical pitch $p$ is normally much larger than the molecular layer thickness and is in general incommensurate with the layer spacing.

In the $\mathrm{Sm} \mathrm{C} \mathrm{C}^{*}$ phase, thermally excited molecular rotation around the long molecular axis is biased. This biasing induces a non-zero in-plane polarization $\boldsymbol{P}(\boldsymbol{r})$ that precesses around the helical axis as one goes from one smectic layer to another.

Most liquid crystal materials that form the Sm $\mathrm{C}^{*}$ phase undergo a second order phase transition to the high tempcrature $\mathrm{Sm} \mathrm{A}$ phase at $T_{\mathrm{c}}$.
In the Sm A phase molecules are still arranged in layers but the tilt angle $\theta$ and the spontaneous in-plane polarization $\boldsymbol{P}$ are equal to zero.

External fields couple strongly to the anisotropies of liquid crystal susceptibilities. In particular, a static magnetic field $\boldsymbol{H}=\left(H_{x}, 0,0\right)$ pcrpendicular to the helical axis $e_{z}$ tends to align the molecules in the direction of the field if the anisotropy of the magnetic susceptibility $\Delta \chi=\chi_{\|}-\chi_{\perp}$ is positive. The helicoidal structure is distorted and the director field $\boldsymbol{n}(\boldsymbol{r})$ changes from a plane-wave structure at $H_{x}=0$ to a soliton-like configuration at $H_{x} \neq 0$. The distortion of the director field $\boldsymbol{n}(\boldsymbol{r})$ is accompanied by an increase of the pitch of the helix. High magnetic fields completely unwind the helical structure and the helicoidal $\mathrm{Sm} \mathrm{C}^{*}$ phase transforms into a uniform $\mathrm{Sm} \mathrm{C}$ phase. It has been predicted a long time ago that a Lifshitz point should exist in the $(H, T)$ phase diagram of a ferroelectric liquid crystal. The Lifshitz point is a special kind of triple point, where the disordered $\mathrm{Sm}$ A phase $(\theta=0, P=0)$, the helicoidally ordered $\mathrm{Sm} \mathrm{C}^{*}$ phase $(\theta \neq 0, \boldsymbol{P} \neq 0, \boldsymbol{q} \neq 0)$ and the homogeneously ordered Sm C phase $(\theta \neq 0, P \neq 0, q=0)$ coexist.

The helicoidal structure of the ferroelectric Sm 
$\mathrm{C}^{*}$ phase also influences the properties of thermally excited orientational fluctuations of the director field $\boldsymbol{n}(\boldsymbol{r})$. These can be decomposed into fluctuations of the magnitude of the tilt angle $\theta$ (amplitudon mode) and fluctuations of the phase of the helical structure $\Phi$ (phason mode). These two modes have been observed experimentally. It has also been shown that the appearance of the helicoidal $\mathrm{Sm} \mathrm{C}^{*}$ phase is the result of "freezing" of a doubly degenerate soft mode with wave vector $q_{c}=2 \pi / p$, which splits below $T_{c}$ into the amplitudon and the phason modes.

The external magnetic field also influences the thermal fluctuations of the director field. It has been shown for similar helicoidal systems that an in-plane magnetic field should induce a gap in the phason dispersion relation at the edge of the Brillouin zone, thus separating the low-frequency acoustic-like phason branch from the high frequency, optic-like branch.

In this paper we shall discuss the phase diagram and the order parameter excitation spectrum of a ferroelectric liquid crystal in a high magnetic field, applied parallel to the smectic layers. The influence of the magnetic field on thermally excited phase fluctuations will be discussed and the theoretical predictions will be compared with the experimental results.

\section{2. $(H, T)$ phase diagram of a ferroelectric liquid crystal}

The phase diagram of a ferroelectric liquid crystal in an external magnetic field can be conveniently derived from the Landau thermodynamic potential $[2,3]$. The $\mathrm{Sm} \mathrm{C}^{*}$ phase can be described by a two-component vector $\boldsymbol{\xi}=$ $\left(\xi_{1}, \xi_{2}, 0\right)$ :

$\xi_{1}=n_{x} n_{z}=\theta \cos \Phi(z)$,

$\xi_{2}=n_{y} n_{z}=\theta \sin \Phi(z)$,

and the in-plane polarization $\boldsymbol{P}$ :

$P_{x}=-P_{0} \sin \Phi(z)$,

$P_{y}=P_{0} \cos \Phi(z)$.
After writing down the invariants with respect to the Sm A phase symmetry and minimizing the free energy [2] with respect to $P_{x}$ and $P_{y}$ one obtains the following free-energy density:

$$
\begin{aligned}
g(z)= & g_{0}+\frac{1}{2} a\left(\xi_{1}^{2}+\xi_{2}^{2}\right)+\frac{1}{4} b\left(\xi_{1}^{2}+\xi_{2}^{2}\right)^{2} \\
& -\Lambda\left(\xi_{1} \frac{\mathrm{d} \xi_{2}}{\mathrm{~d} z}-\xi_{2} \frac{\mathrm{d} \xi_{1}}{\mathrm{~d} z}\right) \\
& +\frac{1}{2} K_{33}\left(\left(\frac{\mathrm{d} \xi_{1}}{\mathrm{~d} z}\right)^{2}+\left(\frac{\mathrm{d} \xi_{2}}{\mathrm{~d} z}\right)^{2}\right)-\frac{1}{2} \Delta \chi H_{x}^{2} \xi_{1}^{2} .
\end{aligned}
$$

Here $a=\alpha\left(T-T_{\mathrm{c}}\right), \alpha$ and $b$ are positive constants, $A$ is the Lifshitz constant and $K_{33}$ is the torsional elastic constant.

The phase transition boundaries $\mathrm{Sm} \mathrm{A} \rightarrow \mathrm{Sm} \mathrm{C}$ and $\mathrm{Sm} \mathrm{A} \rightarrow \mathrm{Sm} \mathrm{C} \mathrm{C}^{*}$ can be determined by linear stability analysis [4]. In the case of positive anisotropy $\Delta \chi>0$, the $\mathrm{Sm} \mathrm{A}$ to $\mathrm{Sm} \mathrm{C}^{*}$ transition temperature $T_{\mathrm{AC}}(H)$ increases monotonically with increasing magnetic field:

$$
\begin{aligned}
T_{\mathrm{AC}}(H) & =T_{\lambda}(H) \\
& =T_{\mathrm{C}}^{\mathrm{R}}+\frac{\Lambda^{2}}{K_{33} \alpha}\left(1+\left(\frac{H}{H_{\mathrm{L}}}\right)^{2}\right)^{2} .
\end{aligned}
$$

Here $H_{\mathrm{L}}$ is the so-called Lifshitz field $H_{\mathrm{L}}=$ $\sqrt{\left(4 \Lambda^{2} / K_{33} \Delta \chi\right)}$ and $T_{\mathrm{C}}^{\mathrm{R}}$ is the phase transition temperature for the racemic material in zero field. The wave vector $q_{c}(H)=2 \pi / p(H)$ of the $\mathrm{Sm} \mathrm{C}^{*}$ phase is temperature independent within this model. It changes continuously along the $\lambda$-line:

$q_{c}(H)=q_{0} \sqrt{1-\left(\frac{H}{H_{\mathrm{L}}}\right)^{4}}$

and equals zero at the Lifshitz field.

In the region above the Lifshitz field, $H>H_{\mathrm{L}}$, the Sm A phase transforms into the homogeneous Sm C phase at the phase boundary given by

$$
T_{\text {AC }}(H)=T_{\mathrm{C}}^{\mathrm{R}}+4 \frac{\Lambda^{2}}{K_{33} \alpha}\left(\frac{H}{H_{\mathrm{L}}}\right)^{2}, \quad q_{\mathrm{c}}(H)=0 .
$$


At the Lifshitz point $\left(H_{\mathrm{L}}, T_{\mathrm{L}}\right)$, the phase boundaries $T_{\lambda}(H)$ and $T_{\mathrm{AC}}(H)$ have a common tangent. The phase transitions $\mathrm{Sm} \mathrm{A} \rightarrow \mathrm{Sm} \mathrm{C}^{*}$ and $\mathrm{Sm} \mathrm{A} \rightarrow \mathrm{Sm} C$ are of second order, whereas the $\mathrm{Sm} \mathrm{C} \mathrm{C}^{*} \rightarrow \mathrm{Sm} \mathrm{C}$ transition has been shown to be of first order near the Lifshitz point [4]. The $\mathrm{Sm} \mathrm{C}^{*} \rightarrow \mathrm{Sm} \mathrm{C}$ transition line is tangential to $T_{\lambda}\left(H_{\mathrm{L}}\right)$ and $T_{\mathrm{AC}}\left(H_{\mathrm{L}}\right)$ at the Lifshitz point.

Far below the $\lambda$-line, the Sm $\mathrm{C}^{*}$ to $\mathrm{Sm} \mathrm{C}$ phase transition boundary can be determined in the constant amplitude approximation [5] $(\theta=$ const.). The phase dependent part of the free energy,

$$
\begin{aligned}
g(\Phi)= & -\Lambda \theta^{2} \frac{\mathrm{d} \Phi}{\mathrm{d} z}+{ }_{2}^{1} K_{33} \theta^{2}\left(\frac{\mathrm{d} \Phi}{\mathrm{d} z}\right)^{2} \\
& -\frac{1}{2} \Delta \chi H_{x}^{2} \theta^{2} \cos ^{2} \Phi(z)
\end{aligned}
$$

leads after minimization to the Sine-Gordon equation

$$
\frac{\mathrm{d}^{2} \Phi}{\mathrm{d} z^{2}}=-\frac{\Delta \chi H^{2}}{2 K_{33}} \sin 2 \Phi .
$$

The phase profiles $\Phi(z)$ that minimize the free energy have the form of a $\pi$-soliton lattice with (a)

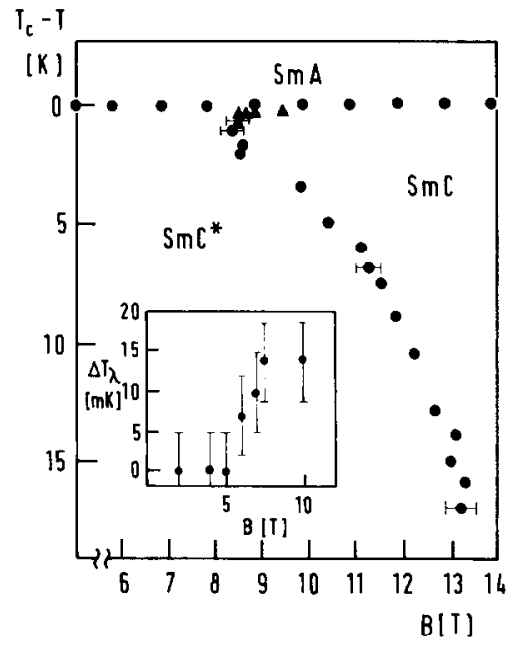

(c)

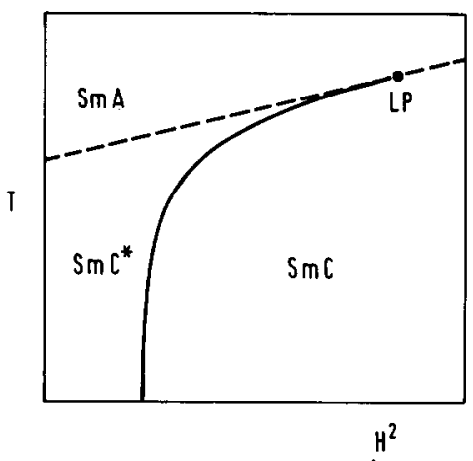

(b)

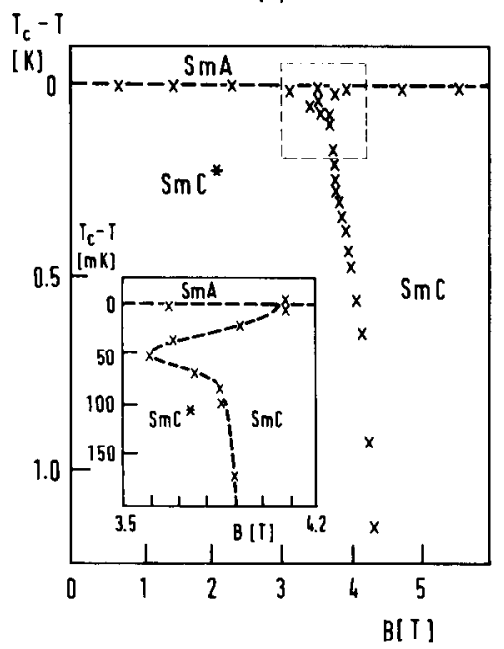

(d)

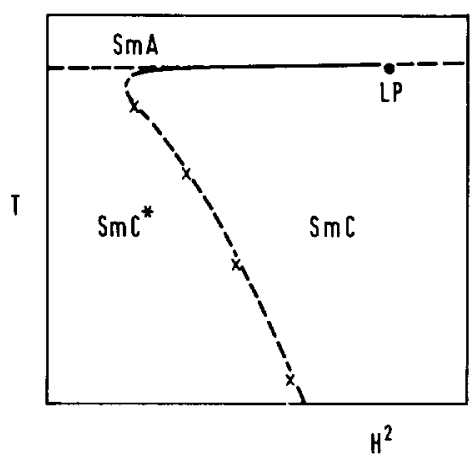

Fig. 1. $(H, T)$ phasc diagram of a ferroelectric liquid crystal DOBAMBC (a) and a mixture of racemic and chiral DOBAMBC (b) as determined by light scattering. The inset to (a) shows magnetic field dependence on the $\lambda$-line. Theoretically predicted $(H, T)$ phase diagrams are shown in (c,d) as derived from a simple [3] and extended form of a free-energy expansion, respectively. 
the field dependent wave vector,

$q_{c}(H)=\frac{\pi}{2} \sqrt{\frac{\Delta \chi}{K_{33}}} \frac{H_{x}}{k K(k)}$,

where $K(k)$ is the complete elliptic integral of the first kind and the parameter $k$ is obtained from

$k=\frac{H_{x}}{H_{c}} E(k)$.

$E(k)$ is the complete clliptic integral of the second kind and the critical field $H_{\mathrm{c}}$ is given by

$H_{\mathrm{c}}=\frac{\pi}{2} \sqrt{\frac{K_{33} q_{0}^{2}}{\Delta \chi}}$

In this model the critical magnetic field for the $\mathrm{Sm} \mathrm{C}^{*}$ to $\mathrm{Sm} \mathrm{C}$ phase is thus temperature independent and is equal to $H_{\mathrm{c}}=\frac{1}{4} \pi H_{\mathrm{L}}$

The above predictions of the simple freeenergy expansion (eq. (3)) are in partial disagreement with available experimental data.
The experimentally determined $(H, T)$ phase diagrams of $p$-decyloxybenzilidene- $p^{\prime}$-amino-2methylbutyl cinnamate (DOBAMBC) [5] and of a mixture of pure and racemic DOBAMBC [6] are shown in fig. $1(\mathrm{a}, \mathrm{b})$, respectively. The phase transition linc $H_{\mathrm{c}}(\mathrm{T})$ shows a strong temperature dependence in both cases and a very narrow region of the re-entrant $\mathrm{SmC}^{*}$ phase was observed near the $\lambda$-line. The appearance of this phase cannot be explained from the free energy expansion in eq. (3). It has also been shown that the product $H(T) \cdot p(T)$ is temperature independent which is an indication that the constant amplitude approximation is valid.

The stability limit of the $\mathrm{Sm} \mathrm{A}$ phase shows a very weak magnetic field dependence. High temperature resolution measurements have shown that the increase of the $\mathrm{Sm} \mathrm{A}$ to $\mathrm{Sm} \mathrm{C}^{*}$ transition temperature is of the order of $10 \mathrm{mK}$ at $10 \mathrm{~T}$ for pure DOBAMBC, as shown in the inset to fig. 1(a). This is in agreement with the predictions of eq. (6) and confirms that the chiral terms in the free-energy expansion represent only a small perturbation. Whereas the Lifshitz point could

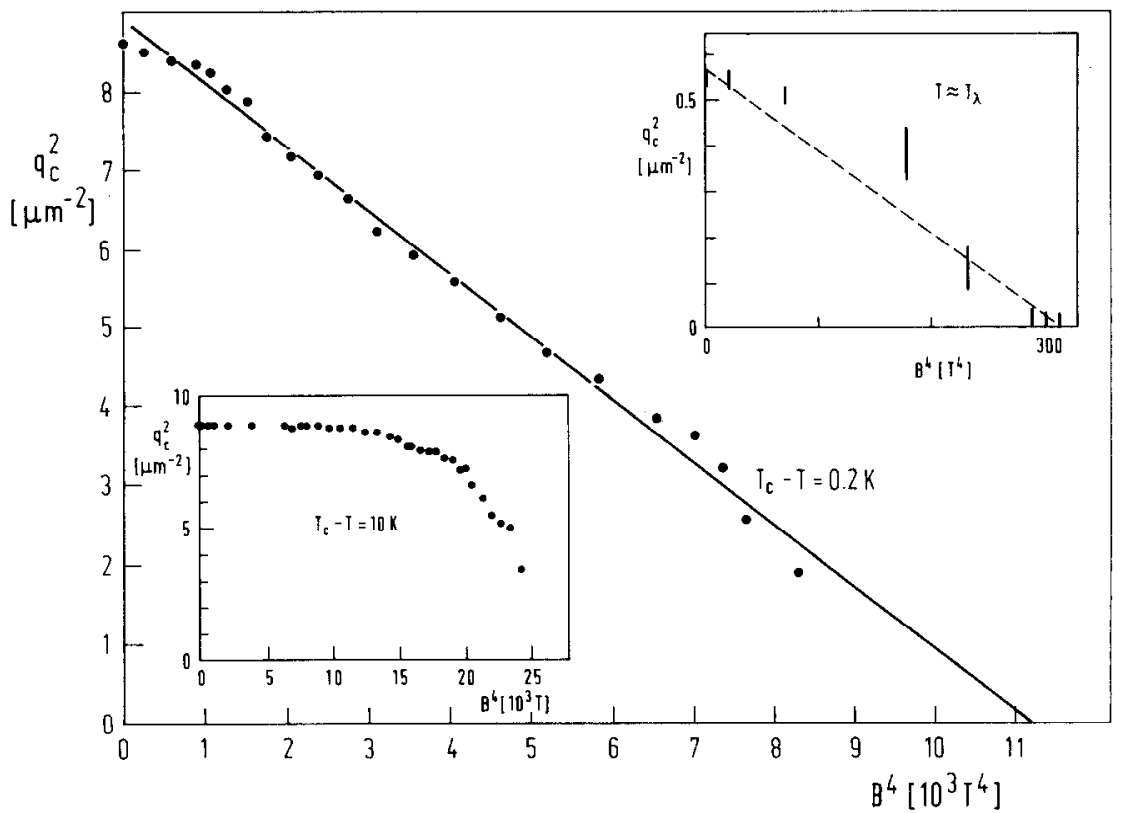

Fig. 2. Magnetic field dependence of the wave vector of the helix in DOBAMBC, indicating the vicinity of the Lifshitz point. At lower temperatures, the unwinding mechanism in the $\mathrm{Sm} \mathrm{C} \mathrm{C}^{*}$ phase of DOBAMBC is different, as shown in the lower inset. The upper inset shows magnetic field dependence of the wave vector of the helix in a mixture of chiral and racemic $D O B A M B C$. 
not be reached in the $(H, T)$ diagram of pure chiral DOBAMBC, it was found in the $(H, T)$ phase diagram of a mixture of five parts of racemic and four parts of chiral DOBAMBC at $H_{\mathrm{L}}=4.16 \mathrm{~T}$ and $T_{\mathrm{c}}-T_{\mathrm{L}} \leqslant 5 \mathrm{mK}$. In both cases a re-entrant $\mathrm{Sm} \mathrm{C} \mathrm{C}^{*}$ phase was observed.

The temperature dependence of the pitch of the helix and the strong temperature dependence of the critical magnetic field can be qualitatively explained within the framework of an "extended" Landau free-energy expansion [7], where coupling terms between the tilt and polarization that describe quadrupolar order of the helical structure are introduced. The pitch of the helix $p(T)$ and the critical magnetic field $H_{c}(T)$ are temperature dependent within the extended model and are in qualitative agreement with the experimental data. The $(H, T)$ phase diagram derived from the extended model is shown in fig. 1(d), whereas fig. 1 (c) shows the $(H, T)$ phase diagram as derived from the simple model (eq. (3)). The shapes of the $\mathrm{Sm} \mathrm{A}$ to $\mathrm{Sm} \mathrm{C}^{*}$ and the $\mathrm{Sm} \mathrm{A}$ to $\mathrm{Sm} \mathrm{C}$ phase boundaries as well as the position of the Lifshitz point are not influenced by these additional terms in the free-energy expansion.

Just below the $\lambda$-line, measurcments of the magnetic field dependence of the wave vector of the helix follow eq. (5), indicating the vicinity of the Lifshitz point in chiral DOBAMBC (see fig. 2 ). At lower temperatures the nature of the $\mathrm{Sm} \mathrm{C}^{*} \rightarrow \mathrm{Sm} C$ phase transition is clearly different, as shown in the lower inset to fig. 2 .

\section{Inelastic light scattering on the $\mathrm{Sm} \mathrm{C}^{*}$ order parameter fluctuations in an external magnetic field}

The dynamics of thermally excited fluctuations of the $\mathrm{Sm} \mathrm{C}^{*}$ order parameter can be derived from the free-energy expansion (eq. (3)). By writing down the non-equilibrium value of the $\mathrm{Sm} \mathrm{C}^{*}$ order parameter as

$$
\begin{aligned}
\xi_{1}(r, t) & -\xi_{1}^{0}+\delta \xi_{1}(r, t) \\
& =\left(\theta_{0}+\delta \theta(z, t)\right) \cos \left(q_{0} z+\delta \Phi(z, t)\right),
\end{aligned}
$$

$$
\begin{aligned}
\xi_{2}(\boldsymbol{r}, t) & =\xi_{2}^{0}+\delta \xi_{2}(\boldsymbol{r}, t) \\
& =\left(\theta_{0}+\delta \theta(z, t)\right) \sin \left(q_{0} z+\delta \Phi(z, t)\right),
\end{aligned}
$$

one can see that any change of the order parameter $\boldsymbol{\xi}(\boldsymbol{r}, t)$ can be written as a linear combination of a small change in the tilt angle magnitude $\delta \theta(z, t)$ and a small phase change $\delta \Phi(z, t)$ :

$$
\delta \xi(r, t)=\delta \theta(r, t) \frac{\xi_{0}}{\left|\xi_{0}\right|}+\delta \Phi(r, t) \frac{e_{z} \times \xi_{0}}{\left|\xi_{0}\right|}
$$

To obtain the dynamics of the order parameter, one has to expand the free-energy density around the equilibrium value in terms of $\delta \theta(z, t)$ and $\delta \Phi(z, t)$. In the case of zero magnctic field, the Landau-Khalatnikov equations of motion for the non-equilibrium value of the order parameter are solved assuming a Bloch expansion for the amplitude and phase fluctuations, propagating along the helical axis. In the Sm A phase one obtains a doubly degenerate soft mode with the relaxation rate

$$
\begin{aligned}
& \tau_{1}^{-1}\left(q_{z}\right)=\frac{K_{33}}{\gamma}\left(q_{c} \pm q_{z}\right)^{2}+\frac{\alpha}{\gamma}\left(T-T_{\mathrm{c}}\right), \\
& T>T_{\mathrm{c}} .
\end{aligned}
$$

Here $\gamma$ is the viscosity of the Sm A phase. Below $T_{\mathrm{c}}$ the degeneracy of the soft mode is removed and we find an amplitudon mode with relaxation rate

$$
\begin{gathered}
\tau_{1}^{-1}\left(q_{z}\right)=\frac{1=33}{\gamma}\left(q_{c} \pm q_{z}\right)^{2}+\frac{2 \alpha}{\gamma}\left(T_{\mathrm{c}}-T\right), \\
T<T_{\mathrm{c}}
\end{gathered}
$$

and a phason mode with the relaxation rate

$\tau_{2}^{-1}\left(q_{z}\right)=\frac{K_{33}}{\gamma}\left(q_{c}-q_{z}\right)^{2}, \quad T<T_{\mathrm{c}}$.

The amplitudon mode rcpresents the fluctuation of the magnitude of the tilt angle and can be observed only very close to $T_{c}$. The phason mode can be considered as a small, plane-wave excitation of the phase of the tilt angle and is temperature independent within this model. 
Dynamic light scattering experiments on DOBAMBC $[8,9]$ have confirmed the above predictions. As is shown in fig. 3, the soft mode has been observed in the $\mathrm{Sm} A$ phase of DOBAMBC. It "freezes out" at the phase transition temperature $T_{\mathrm{c}}$. In the $\mathrm{Sm} \mathrm{C}$, two modes have been observed and have been identified as the amplitudon and the phason mode. These experimental results clearly indicate that the soft mode concept can be applied to the $\mathrm{Sm} \mathrm{A} \rightarrow \mathrm{Sm} \mathrm{C}^{*}$ phase transition.

An external magnetic field, applied parallel to the smectic layers, influences the static and dynamic properties of the $\mathrm{Sm} \mathrm{C} \mathrm{C}^{*}$ order parameter. In the low temperature limit one can use a constant amplitude approximation and we can discuss the effect of the magnetic field on the phason mode, neglecting amplitude changes. In this case, the $\mathrm{Sm} \mathrm{C}^{*}$ order parameter is written as

$$
\begin{aligned}
& \xi_{1}(z, t)=\theta_{0} \cos \left(\Phi_{0}(z)+\delta \Phi(z, t)\right), \\
& \xi_{2}(z, t)=\theta_{0} \sin \left(\Phi_{0}(z)+\delta \Phi(z, t)\right),
\end{aligned}
$$

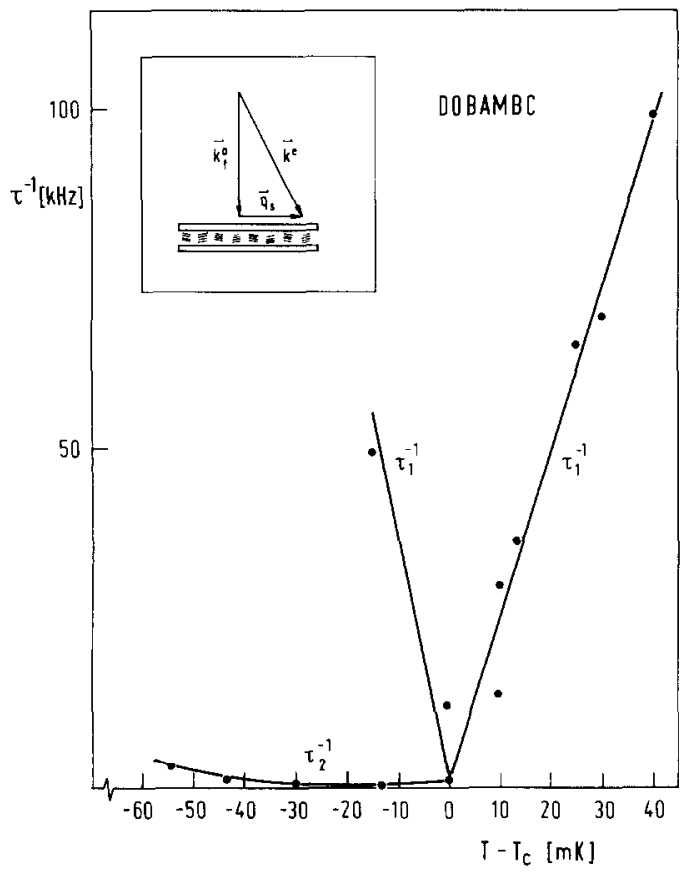

Fig. 3. Temperature dependence of order parameter relaxation rates at the $\mathrm{Sm} \mathrm{A} \rightarrow \mathrm{Sm} \mathrm{C} \mathrm{C}^{*}$ transition. The scattering wave vector is equal to $q_{c}\left(T=T_{\mathrm{c}}\right)$.
After expanding $\delta \Phi(z, t)$ in the Bloch form,

$\delta \Phi(z, t)=\sum_{q} \Phi_{q}(z) \exp \left(-\frac{t}{\tau(q)}\right)$,

and remembering that $\Phi_{0}(q, H)$ is a solution of the Sine-Gordon equation, one obtains Lame's equation of order one [10] for the Bloch functions $\Phi_{q}(z)$ :

$\frac{\mathrm{d}^{2} \Phi_{q}}{\mathrm{~d} u^{2}}+\left[h-2 k^{2} s n^{2}(u, k)\right] \Phi_{q}=0$.

Here $u=z / \beta k$ is an argument of a Jacobian elliptic function of modulus $k, h=k^{2}\left[\beta^{2} \gamma \tau(q) /\right.$ $\left.K_{33}+1\right]$, and $\beta^{2}=K_{33} /\left(\Delta_{\chi} H^{2}\right)$ is a magnetic coherence length. Solutions of eq. (17) have been discussed in connection with the dynamics of chiral nematics in an external magnetic field $[10,11]$. It has been shown that for certain limiting values of the wave vector $q_{z}$ the relaxation rates of the phason mode $\tau_{2}^{-1}\left(q_{z}\right)$ can be written as

$$
\begin{aligned}
\tau_{2}^{-1}\left(q_{z}\right) & \simeq \frac{K_{33}}{\gamma} \frac{\left(1-k^{2}\right) K^{2}(k)}{E^{2}(k)}\left(q_{c} \pm q_{z}\right)^{2}, \\
q_{z} & \approx q_{c}, \\
\tau_{2-}^{-1}\left(q_{z}\right) & \simeq \frac{K_{33}}{\gamma} \frac{1-k^{2}}{\beta^{2} \frac{k^{2}}{2}}, \quad q_{z} \leqslant 2 q_{z}, \\
\tau_{2+}^{-1}\left(q_{z}\right) & \simeq \frac{K_{33}}{\gamma} \frac{1}{\beta^{2} k^{2}}, \quad q_{z}
\end{aligned}
$$

The effect of an external magnetic field on the phason dispersion is shown in fig. 4. The phason branch splits into an optic-like $\tau_{2+}^{-1}(q)$ and an acoustic-like branch $\tau_{2-}^{-1}(q)$, separated by a field dependent frequency gap $\Delta G(H)=\tau_{2+}^{-1}\left(2 q_{c}\right)-$ $\tau_{2-}^{-1}\left(2 q_{c}\right)$ at the wave vector $2 q_{c}(H)$, corresponding to the edge of the Brillouin zone. The magnetic field dependence of the gap is shown in the inset to fig. 4. It should be stressed that the gap appears in the regime, where the pitch of the helix and the corresponding wave vector are not influenced by the field. It is therefore a direct consequence of a cross-over from the plane-wave to the soliton-like dynamics of the $\mathrm{Sm} \mathrm{C}^{*}$ order parameter. 


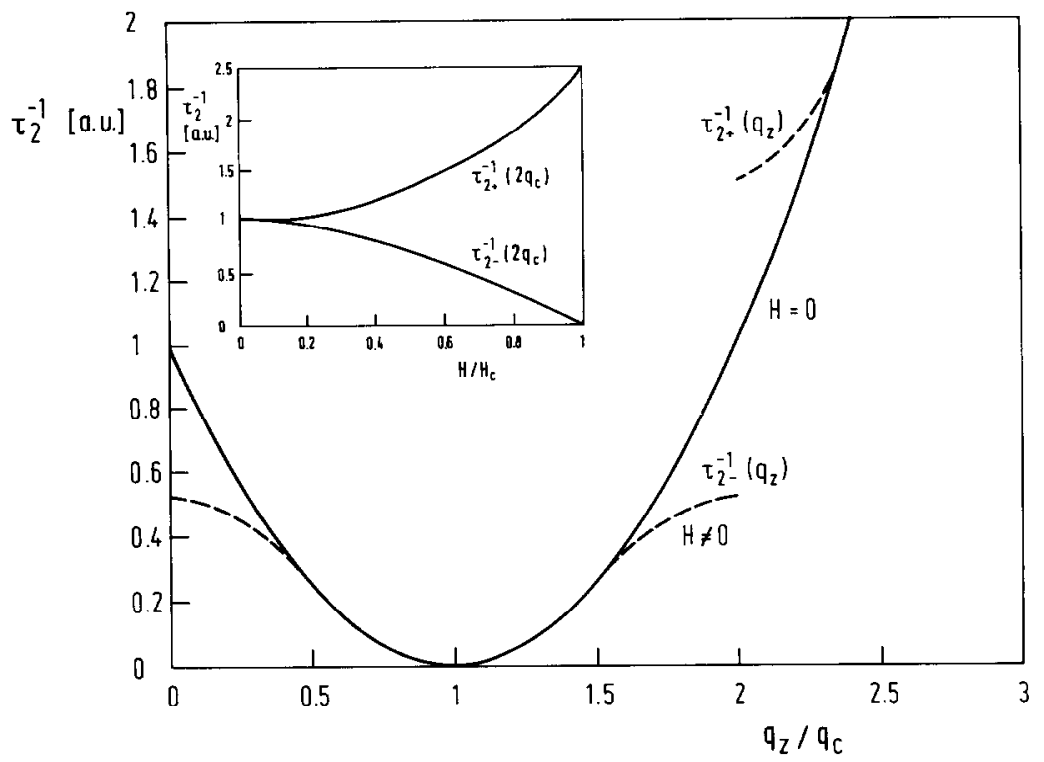

Fig. 4. Magnetically induced band-structure of the phason dispersion branch. The inset shows magnetic field dependence of the splitting of the phason branch at the edge of the Brillouin zone $q_{z}=2 q_{c}$.

Angular fluctuations of the $\mathrm{Sm} \mathrm{C}^{*}$ director $\boldsymbol{n}(\boldsymbol{r}, t)$ are directly coupled to the fluctuations of the dielectric tensor $\delta \varepsilon(r, t)$ and are responsible for strong depolarized scattering of light in the Sm $C^{*}$ phase. In a dynamic light scattering experiment, one measures the autocorrelation function $G\left(t^{\prime}\right)$ of the scattered light intensity at a certain wave vector

$\boldsymbol{q}_{\mathrm{s}}=\boldsymbol{k}_{\mathrm{i}}-\boldsymbol{k}_{\mathrm{s}}$.

Here $\boldsymbol{k}_{\mathrm{i}}$ designates the wave vector and polarization of the incident beam, and $\boldsymbol{k}_{\mathrm{s}}$ denotes the wave vector and polarization of the scattered beam. In the Born approximation, the autocorrelation function $G\left(t^{\prime}\right)$ of the scattered light intensity is proportional to the autocorrelation function of the projection of the fluctuating part of the dielectric tensor on the incoming and scattered light polarizations at the scattering wave vector $q_{\mathrm{s}}$ :

$G\left(t^{\prime}\right) \propto\left\langle\epsilon_{\mathrm{is}}\left(q_{\mathrm{s}}, 0\right) \epsilon_{\mathrm{is}}^{*}\left(q_{\mathrm{s}}, t^{\prime}\right)\right\rangle$.

It can be shown [9] that off-diagonal terms of the fluctuating part of the dielectric tensor $\delta \epsilon(r, t)$ are directly proportional to the fluctuating part of the order parameter $\delta \boldsymbol{\xi}(\boldsymbol{r}, t)$. In particular,

$\delta \epsilon_{x z} \propto \delta \xi_{1}$ and $\delta \epsilon_{y z} \propto \delta \xi_{2}$.

The fluctuations of the order parameter $\delta \xi(r, t)$ are thus observable in a scattering geometry, where an incoming beam is ordinarily polarized and the scattered beam is extraordinarily polarized or vice versa.

Recently [12] a light scattering experiment in a magnetic field was performed in the $\mathrm{Sm} \mathrm{C}^{*}$ phase of a mixture of $35 \%$ of a pure ferroelectric liquid crystal 4-(2'-methylbutyl)phenyl $\quad 4^{\prime}$-n-octylbiphenyl-4-carboxylate (CE-8) and $65 \%$ of racemic liquid crystal CE-8R. The dispersion of the phason mode has been measured by the photon autocorrelation technique at different magnetic fields up to $12 \mathrm{~T}$. Dispersion relations for the phason mode, measured at 0 and $6 \mathrm{~T}$, are shown in fig. 5 . It can be clearly seen that the external magnetic field significantly changes the shape of the phason dispersion. Numerical analysis shows an approximate $20 \%$ decrease of the effective damping constant $K_{33} / \gamma$ at $6.65 \mathrm{~T}$, 


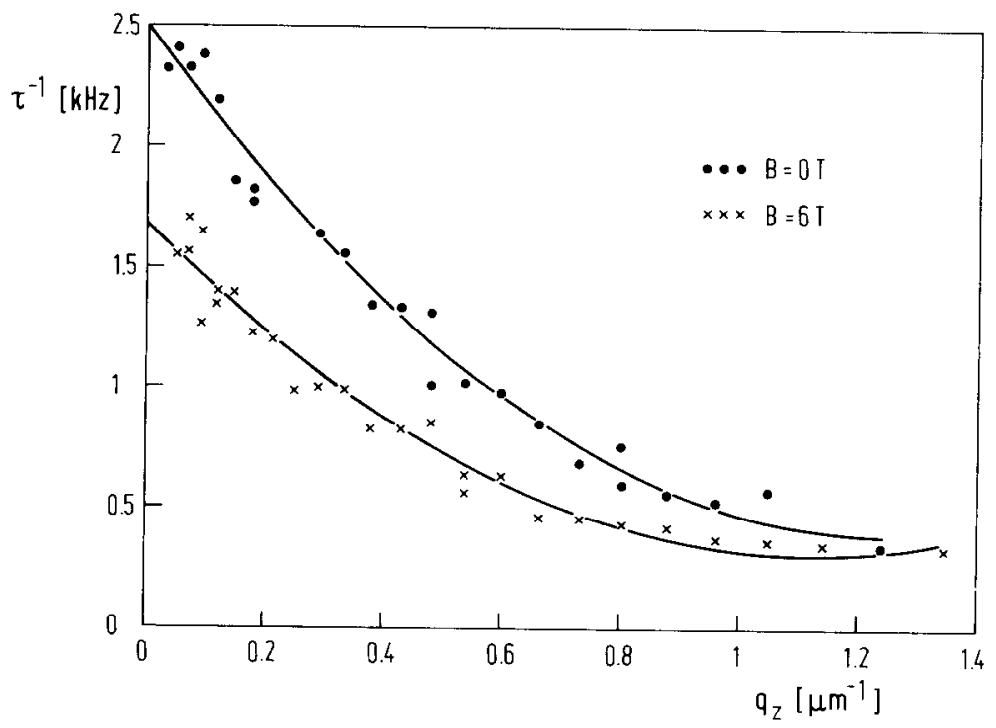

Fig. 5. Phason dispersion as measured in a mixture of chiral and racemic CE-8 at 0 and $6.0 \mathrm{~T}$. (Note: these results contain a small

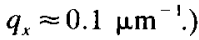

which is in agreement with eq. (18). Extrapolated values of the relaxation rate $\tau_{2-}^{-1}(q \rightarrow 0)$ show a large decrease, indicating the appearance of a gap in the phason dispersion relation. It should be stressed that the observed changes cannot be attributed merely to the change of the pitch of the helix, as the pitch of the helix is almost unaffected at these values of the magnetic field.

These preliminary results clearly indicate that in the $\mathrm{Sm} \mathrm{C} \mathrm{C}^{*}$ phase, phase fluctuations show a cross-over between the plane-wave and the soliton-like dynamics in agreement with theory.

We may conclude that the dynamics of the Sm $\mathrm{C}^{*}$ order parameter is well described within the framework of a simple Landau thermodynamic potential, whereas the phase diagram of a ferroelectric liquid crystal in high magnetic fields can be described by an extended form of the Landau thermodynamic potential.

\section{References}

[1] R.B. Meyer, L. Liebert, L. Strzelecki and P.J. Keller, J. Phys. Lett. 36 (1975) L69.

[2] S.A. Pikin and V.L. Indenbom, Usp. Fiz. Nauk 125 (1978) 251.

[3] R. Blinc and B. Žeks, Phys. Rev. A 18 (1978) 740.

[4] A. Michelson, Phys. Rev. Lett. 39 (1977) 464.

[5] I. Muševič, R. Žekš, B. Blinc, Th. Rasing and P. Wyder, Phys. Rev. Lett. 48 (1982) 192.

[6] A. Seppen, Ph.D. Thesis, University of Nijmegen (1987).

[7] B. Urbanc and B. Žekš, Liquid Crystals 5 (1989) 1075.

[8] I. Muševič, R. Blinc, C. Filipič, T. Copič, A. Seppen, P. Wyder and A. Levanyuk, Phys. Rev. Lett. 60 (1988) 1530.

[9] I. Drevenšek, I. Muševič and M. Čopič, Phys. Rev. A 41 (1990) 923.

[10] J.D. Parsons and C.F. Hayes, Phys. Rev. 9 (1974) 2652.

[11] C. Fan, L. Kramer and M.J. Stephen, Phys. Rev, A 2 (1970) 2482.

[12] I. Muševič, B. Žekš, R. Blinc, H.A. Wierenga and Th. Rasing, Physica B 177 (1992) 511 (these Proceedings). 